\title{
IDENTIFYING BEST TREATMENT FOR DISEASE IN EXTRACTION OF MEDICAL RELATION USING MACHINE LEARNING APPROACH
}

\author{
DhanwatePooja $\mathbf{S}^{\mathbf{1}}$, UbaleRavina $\mathbf{R}^{\mathbf{2}}$, KatharPriyanka $\mathbf{U}^{\mathbf{3}}$, Kapadnis Swati $\mathbf{J}^{\mathbf{4}}$ \\ ${ }^{I}$ Student, Computer Engineering, SND college of EngineeringYeola,SPPU, Maharashtra, India \\ ${ }^{2}$ Student, Computer Engineering, SND college of EngineeringYeola,SPPU, Maharashtra, India \\ ${ }^{3}$ Student, Computer Engineering, SND college of EngineeringYeola, SPPU, Maharashtra, India \\ ${ }^{4}$ Student, Computer Engineering, SND college of EngineeringYeola,SPPU, Maharashtra, India
}

\begin{abstract}
dhanwatepuja@gmail.com,ravinaubale03@gmail.com,priyankakathar03@gmail.com,swatikapadnis36@gmail.com
Guided By: Prof. Kumbharde M.V.,Assistant Prof.Computer Engineering, SND college of EngineeringYeola, SPPU, Maharashtra, India
\end{abstract}

\begin{abstract}
Our software has two modules include in it extraction of data \& EHR system with cloud. Now a days there is a busy schedules of doctors so they have no time to read the huge amount of articles, they discussed the disease related problems either in conference or in the seminar only. So we provide a great extraction approach for this problem to give huge information of articles to the system, the machine learning process is done on the article. It only give the information to the doctors which is they want \& related and useful to their work. The second approach includes in our system is that we proposed the EHR(Electronic Health Record) system. Which includes the details information of the patient ex: patient name, id, address ,photo etc. This data also available on cloud. This information is easily accessible and it requires the less time to be searched the data about disease \& treatment. Using the EHR system we can access the medical history of the patient from any location. It reduces the time require for the lab tests, previous treatments, allergies of patient.
\end{abstract}

Keywords: Machine learning, Extraction of data, EHR, Secure storage. Government role, Cloud.

\section{INTRODUCTION}

Now a days people are very careful about their health and want the information about the health related problems. Doctors are also have very busy schedule. If any new research is done in medical field then this data available in large article or in the conference of doctors. Many of them are not attain it because of busy schedule. Traditional way of the treatment is apply to the patient. So the machine learning approach is very useful in this condition. The article is learned by the system $\&$ only the data which the doctor want is provided to them. So the data mining process is done.

Second problem is that it is impossible for doctors to have the record of every patient in a documentary way. The records are maintain easily in the electronic way. Electronic Health Record(EHR) it has becoming the most important feature for storage of medical data. It gives the more reliable approach and the data is easily accessible. It has data related to cure, prevention, side effects, symptoms, previous treatment, allergies of the patient. This data is store on cloud using that we can access it from any where in world. Using this data doctors can take faster decisions for applying the best treatment to patient. The id is provided to the every patient so only the doctor \& patient can access the data. So it is time efficient, secure, easy to maintain, faster process.
It also has one most important part that is Government. All the data which is in the EHR system is also accessible to the government. If any disaster is come then there is most important need of blood to the people. At this situation the price of blood in the blood bank is increase. So the medical data of peoples is available to government also. If there is need of particular blood group then government can convince the people which have the blood group.

\section{RELATED WORK}

In Machine Learning Approach for Identifying DiseaseTreatment Relations in Short Texts By author OanaFrunza ,DianaInkpen, Thomas Tran. at [1]. It involves how the machine learning process is done on the articles which are related to the the medical field. It gives the clear idea about the automatic learning of large articles and extract only the require data.

In Disease Inference from Health-Related Questions via Sparse Deep Learning at[2]. By author LiqiangNie, Meng Wang, Luming Zhang, Shuicheng Yan, Bo Zhang, Tat-Seng Chua. In this paper in give the Health Tape feature for the people which have questions about there health related problems. The solutions are also given in form of answer by doctors. They give detail information about the cure, prevention, best treatment for the disease.

In Review on Machine Learning Approach for Identifying 
Disease Treatment in Short Texts at[3]. By author In this paper they review on the machine learning approach for the data which is in the large article form. How the learning process is done. The algorithms use for it, its features are also given in it.

In Journal "To identify Disease Treatment Relationship in Short Text Using Machine Learning \& NLP" at[4]. In these it describe that how the natural processing is useful for the extraction of data which is in the large volume.

In journal "Efficient Machine Learning for Identifying Disease-Treatment Semantic Relation from Bio-Medical Sentences."at[5]. It give all the clear idea about the medical data which is in the large size. It identify the semantic relation that is present between the query or the data which the user require.

\section{SURVEY EXTRACTION}

We refer various IEEE papers, journals for the survey of topic. From that we study that the system first authenticate the user by using UID. If the user is valid to access the data then \& then only user can access the data otherwise exit from the process. In machine learning process all the data is learned automatically \& mining process on that data is done. Using that only the require data is provided to the user in structural way. In previous system the EHR is available only for limited area. In proposed system we remove these limitation by using cloud concept. Using cloud we access the data from anywhere in the world.

\section{EXISTING SYSTEM}

The information about the medical research is available in the large articles. There is a need of extract the data which is in the article. The data is given which only the doctor want. Not the unwanted data is display in the output. The information of disease is given. All the medical data related to that disease such as symptoms, medicines, newly suggested treatments, methodology use for the treatment. It extract the data from the article and provide it in a structural and suitable way.

\section{PROPOSED SYSTEM}

\subsection{Extraction process in less time:}

Proposed system also have the extraction feature of the data which is related to the medical document. But it gives the faster processing of the document. Provide the data in structural way. From large set of information it mines the data only which is require by the doctor. The research is done on various disease and the new way of treatment is provided in it. It has all the detail data about that disease, its treatment, symptoms, medicines related to that disease, new methodology of treatment by various doctors, its results, affect on the forbearing of that treatment, time require for recovery from that disease. All the data is available in it. But If doctor want information about particular disease then it is easy task for them using the extraction approach of machine learning. The files are in the any form. The require data is extract from it and given to the doctor in suitable and faster way.

\subsection{EHR System with Cloud:}

In this the Electronic Health Record system is provided with the cloud. Because of that we can access the data from EHR from any where in the world. This system contain all the medical history of the forbearing. Which is the previous treatment of forbearing, which doctor treat, date of the treatment, allergies, lab test results, Symptoms of particular disease. Which treatment is suitable for the forbearing, by giving which medicines forbearing recovery is done faster. All the information is given to the doctor. By using it doctor can make faster decisions about the treatment. Using this the less time is require for analyzing the all medical history of forbearing. It also provide the security for the data. One unique id is provided to every forbearing. Using these only the doctor and the forbearing can have the information. The id is given in the from of name, adhar card number, mobile number, photo. So it can give the faster, secure access to forbearing medical history which is useful for taking the fast medical decisions.

\subsection{Government Sector:}

This approach is most useful for the government also. All the record stored in the EHR is also accessible to the government. Using HER government can access the id of forbearing. Using that id all the medical data stored in the electronic health record is accessible to the government also. If any disaster is come then there is most need of blood to the people. At that time price of blood in blood bank is very high. Because of that many of peoples are not able to take it. The record is also available to the government so that it also have the information about the blood group. If there is need of particular blood group then government can convince the people those have that blood group.

\section{SYSTEM ARCHITECTURE}

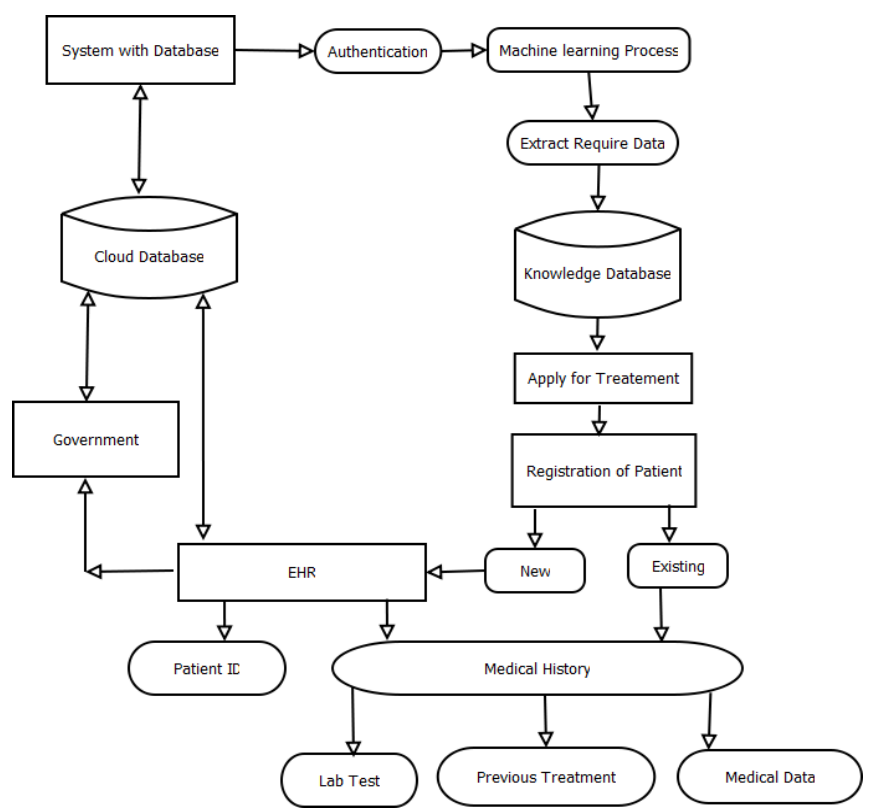

Fig -1: System Architecture 
In this System architecture, there are various components that are described as follows:-

\subsection{EHR System}

In these component of system all the medical data of patient is stored. All the information about lab tests, medical data, previous treatment is stored in it. It has the patient Id for the authentication purpose. Using these security is maintain.

\subsection{Machine Learning Process}

In these component of the system the article which is given to system as a input is learned in machine learning approach . Automatically the all the input file is learned by the system. Only the require data is given to the user.

\subsection{Knowledge Database}

In these component only the require data is collected from the large amount of files after applying the machine learning process on it. Only the require information about the query is shown to user in a structural way.

\subsection{Cloud Database}

Using cloud database we can access the data from anywhere in the world. All the data which is available in HER system is also available in the cloud. Using that the accessing process is done faster and in easy way.

\subsection{Government}

One of the important role in these system is of the government. Government also have all the data of the patient. It is mostly use when any disaster is come. All the medical data is available for the government.

\section{CONCLUSION}

Usingthese system we can have several features. The faster access, secure storage of information. The system useful for doctor, patient, government also. All the medical history, all health related information can be access easily using the HER system. So there is solution provided for two problems related to the medical field. First for the accessing the newly researched medical data using machine learning approach of extraction feature. Second maintain the medical record of the patient and access it from anywhere in the world. Also the security provided for the stored data and access it in very easy way. There is use of cloud because of that the accessibility feature is increase to the doctor, patient, government. All the time require for the lab tests, knowing about previous treatment is reduce. So It is very useful for taking the medical decisions in very fast way. For implementing this system it is a research work in documentation in phase-1 \& still we are working on implementations of actual system in phase- 2 .

\section{ACKNOWLEDGEMENT}

I wish to express my sincere thanks and deep sense of gratitude to respected guide Prof. M. V. Kumbharde in
Department of Computer Engineering of SND college of Engineering \& Research center, Babhulgaon, Yeola 423401, for the technical advice, encouragement and support us \& guide for the guidance about project related queries.

\section{REFERENCES}

[1]. “A Machine Learning Approach for Identifying Disease Treatment Relations in Short Texts." (IEEE Transactions on Knowledge and Data Engineering, vol.23 no.6 27 Aug 2010)

[2]. "Disease Inference from Health Related Questions via Sparse Deep Learning ." (IEEE Transactions on knowledge and data Engineering May 2014)

[3]. "Review on Machine learning Approach for Identifying Disease Treatment Relation in Short Texts".( International Journal of Innovative Research in Advanced Engineering (IJIRAE) Volume 1 Issue 3 (May 2014) SPECIAL ISSUE)

[4]. "Identify Best Treatment For Disease Using Machine Learning Approach In Relation to Short Text."( IRAJ International Conference-Proceedings of ICRIESTAICEEMCS, 29th December 2013, Pune India. ISBN: 978-93-82702-50-4)

[5]. "Efficient Machine Learning For Identifying DiseaseTreatment Semantic Relations from Bio-Medical Sentences."( International Journal Of Computational Engineering Research (ijceronline.com) Vol. 2 Issue. 5)

[6]. "Health Analysis Code System Using Machine Learning.'(PravinShinde et al, / (IJCSIT) International Journal of Computer Science and Information Technologies, Vol. 5 (3) , 2014, 3928-3933)

[7]. "Efficient Extraction of Medical Relation of using Machine Learning Approach."(Volume 3, Issue 3, March 2013 ISSN: 2277 128X International Journal of Advanced Research in Computer Science and Software Engineering)

[8]. "Mining Electronic Health Records Using Machine Learning Approach"(International Journal of Advanced Information Science and Technology (IJAIST) ISSN: 2319:2682 Vol.28, No.28, August 2014)

[9]. "Electronic Health Records: Applications, Techniques and Challenges"'(International Journal of Computer Applications (0975 - 8887) Volume 119 - No.14, June 2015) 